\title{
Le système de pompage air-lift et la remontée des nodules polymétalliques marins
}

\author{
Air-lift pumping and raising \\ of polymetal nodules from the bottom of the sea \\ Professeur Michel Giot \\ Université Catholique de Louvain \\ Département Thermodynamique et Turbomachines
}

\section{Liste des symboles}

$A \quad$ aire de la section droite

$C_{0}, C_{0}^{\prime}$ paramètre de distribution (eq. 4 et 11 )

$D$ diamètre de conduite

$f \quad$ coefficient de frottement

$g$ accélération de la pesanteur

$h_{s} \quad$ longueur du tronçon $G-L$ ou $G-L-S$ immergé

$h_{d} \quad$ longueur du tronçon situé au-dessus du niveau

$$
\text { libre }
$$

$L$ longueur de conduite pour l'écoulement $G-L$ ou $G-L-S$

$L^{\prime} \quad$ longueur de conduite pour l'écoulement $L$ ou $L-S$

$\dot{M} \quad$ débit massique

$p \quad$ pression

$Q \quad$ débit volumique

$R \quad$ constante des gaz parfaits

$T$ température

$w \quad$ vitcssc

$w_{G, j} \quad$ vitesse de glissement de la phase gazeuse

$w_{S, j}$ vitesse de décantation des éléments solides

fraction volumique

rendement

rendement du pompage proprement dit

rendement de la compression

coefficient de perte de charge

masse volumique

rapport d'immersion $\left(\sigma \triangleq h_{S} / L\right)$

\section{Indices}

$\begin{array}{ll}a & \text { conditions atmosphériques } \\ f & \text { frottement } \\ G & \text { gazeux }\end{array}$

$\begin{array}{ll}i & \text { injection } \\ L & \text { liquide } \\ r & \text { refoulement } \\ S & \text { solide } \\ - & \text { moyen } \\ & \text { idéal ou diphasique } L-S\end{array}$

\section{Introduction}

La collecte des nodules de manganèse des fonds océaniques est une entreprise exaltante dans ses aspects techniques. En effet, les échelles en présence nécessitent des efforts peu communs : les profondeurs sous-marines envisagées se situent entre 4000 et $5000 \mathrm{~m}$, les sites miniers sont fort éloignés des côtes, les débits à obtenir se chiffrent en millions de tonnes par an, et... les nodules, petits cailloux noirâtres dont le diamètre dépasse rarement les $10 \mathrm{~cm}$ adhèrent à une couche superficielle parsemée d'embûches.

Diverses méthodes de remontée des nodules ont été essayées ou simplement imaginées : elles sont soit mécaniques, soit hydrauliques. Ne considérant que les procédés hydrauliques schématisés à la figure 1 , nous citerons :

- le système à chambre immergée (a) qui comporte un réservoir maintenu à la pression atmosphérique à quelques $500 \mathrm{~m}$ de profondeur ; le mélange eaunodules est aspiré par la conduite verticale aboutissant dans ce réservoir ; une partie du débit d'eau est refoulée dans l'océan au niveau du réservoir, tandis que le mélange à plus forte concentration est pompé vers la surface par des moyens classiques ;

- le système à pompes immergées (b) :

- le système à pompe en surface (c) : 


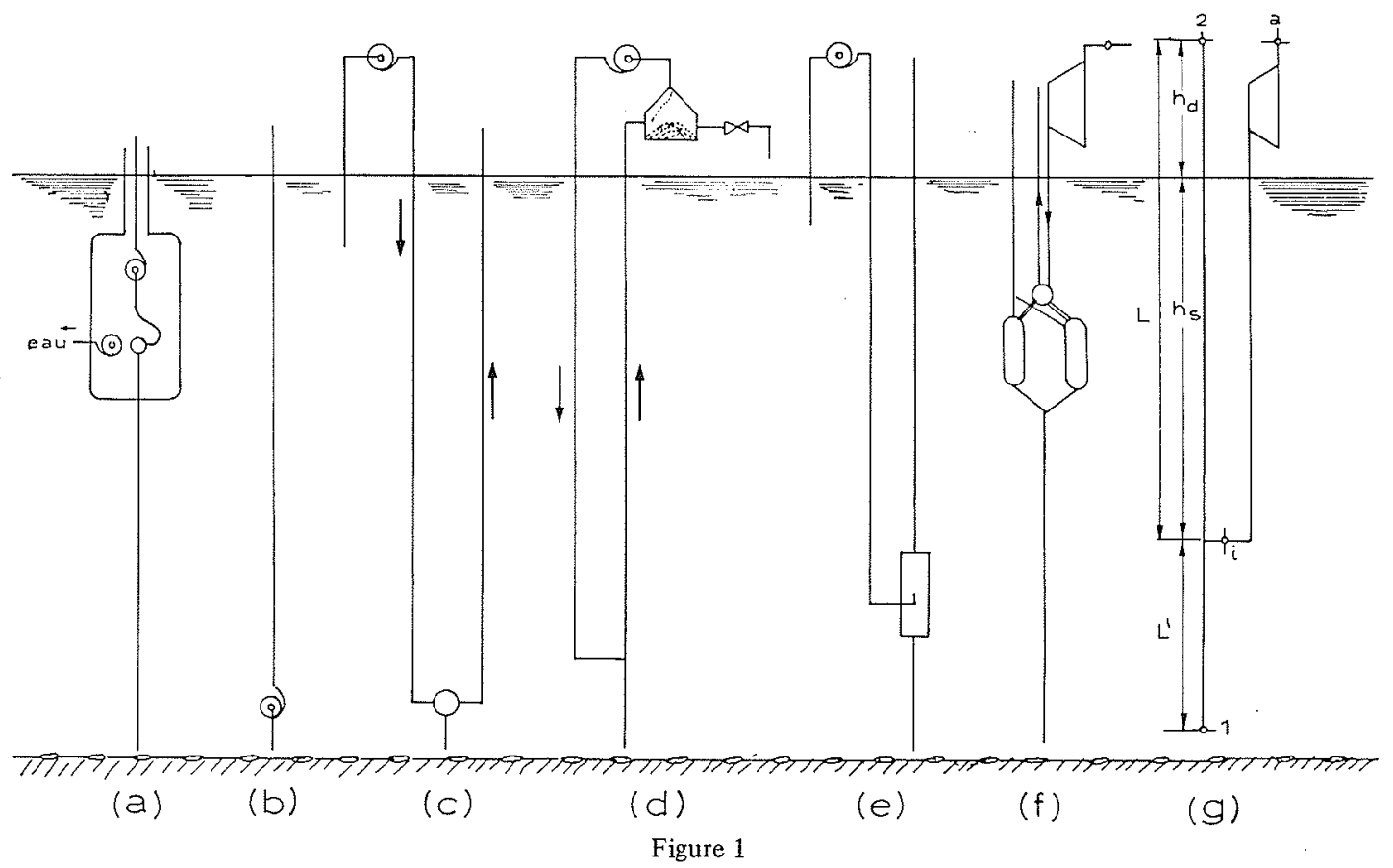

- le système à billes en matière plastique ayant une masse volumique inférieure à celle de l'eau (d) ; la séparation billes-nodules se fait dans un réservoir sous pression en surface (Grabow, 1977);

- le système à éjecteur (e) ;

- le système à pompe PNEUMA (f) ; dans une des versions de ce système, de l'air comprimé est introduit dans une des trois chambres constituant les trois corps de pompe, via un distributeur ; sous l'effet de la pression d'air, le corps de pompe se vide du mélange eau-nodules dans la conduite de refoulement vers le navire minier ; l'air est alors détenđu à la pression atmosphérique, tandis que le mélange eaunodules est admis dans le corps de pompe en provenance de la conduite de dragage par une soupape anti-retour ;

- le système à air-lift $(\mathrm{g})$.

Compte tenu de la longue énumération ci-dessus, l'objet de ce mémoire paraîtra bien modeste puisqu'on tentera simplement ici d'expliquer comment se conçoit et se dimensionne le système à air-lift. Il s'agit toutefois d'un procédé très prometteur depuis le 30 juillet 1970 date à laquelle la Société américaine Deepsea Ventures réussit à l'utiliser avec succès dans l'Océan Atlantique pour pomper des nodules à environ $850 \mathrm{~m}$ de profondeur.

L'exposé est divisé en deux parties. La première concerne le pompage de liquides par air-lift. Les avantages et les inconvénients de ce procédé sont bien connus : au nombre des inconvénients, on cite surtout le rendement assez faible, tandis qu'on retient parmi les avantages : l'absence de moteur immergé, de contact fluide-rotor, la résistance à la corrosion, à l'abrasion, etc.

La seconde partie traite du pompage d'éléments solides par air-lift. Elle est basée sur des informations très récentes, citées en référence et qui inaugurent un nouveau domaine de la mécanique des fluides : celui des fluides triphasiques.

\section{Le pompage de liquides par air-lift}

\section{Détermination de la relation entre le débit de liquide et le débit d'air}

Comme Todoroki et al. (1973), intégrons l'équation de la quantité de mouvement dans le volume de contrôle défini par les sections d'entrée 1 et de sortie 2 de la conduite de pompage, et la paroi de celle-ci. Il vient :

$$
\begin{aligned}
& p_{1}+\rho_{L} w_{L, 1}^{2}= \\
& \quad=p_{a}+\rho_{L} g L^{\prime}+\int_{i}^{2}\left(\alpha_{G} \rho_{G}+\alpha_{L} \rho_{L}\right) g d z+ \\
& +\alpha_{G, 2} \rho_{G, 2} w_{G, 2}^{2}+\alpha_{L, 2} \rho_{L} w_{L, 2}^{2}- \\
& \quad-\int_{1}^{2}\left(\frac{d p}{d z}\right) d z-\int_{f, L}^{2}\left(\frac{d p}{d z}\right)_{f, L G} d z
\end{aligned}
$$

Dans cette équation, $\alpha_{G}$ et $\alpha_{L}$ désignent la fraction de la section droite occupée en moyenne respectivement par les phases gazeuse et liquide $\left(\alpha_{G}+\alpha_{L}=1\right)$; les deux derniers termes sont relatifs aux frottements dans les tronçons mono et diphasiques.

De plus, on a:

$$
p_{1}=p_{a}+\rho_{L} g\left(h_{s}+L^{\prime}\right)-\xi_{e} \frac{\rho_{L} w_{L, 1}^{2}}{2}
$$

le coefficient de perte de charge $\xi_{e}$ dépendant de la géométrie à l'aspiration. Remplaçons $p_{1}$ dans l'équation (1) par son expression (2); on trouve :

$$
\begin{aligned}
\rho_{L} g h_{s} & -\int_{1}^{2}\left(\alpha_{G} \rho_{G}+\alpha_{L} \rho_{L}\right) g d z= \\
& =-\int_{1}^{i}\left(\frac{d p}{d z}\right) d z-\int_{i, L}^{2}\left(\frac{d p}{d z}\right)_{f, L G} d z+ \\
& +\frac{\dot{M}_{G}^{2}}{A^{2} \alpha_{G, 2} \rho_{G, 2}}+\frac{\dot{M}_{L}^{2}}{A^{2} \rho_{L}}\left(\frac{1}{\alpha_{L, 2}}+\frac{\xi_{e}}{2}-1\right)
\end{aligned}
$$


Le premier membre de cette équation est le terme hydrostatique moteur. Notons qu'il peut s'écrire : $\rho_{L} g h_{s}-\int_{i}^{2}\left(\alpha_{G} \rho_{G}+\alpha_{L} \rho_{L}\right) g d z=$
$\quad=\int_{i}^{2} \alpha_{G}\left(\rho_{L}-\rho_{G}\right) g d z-\rho_{L} g h_{d}$ Le taux de vide $\alpha_{G}$ peut s'exprimer en fonction des débits par la méthode de Zuber et Findlay (1965):

$$
\frac{Q_{G}}{\alpha_{G} A}=C_{0} \frac{Q_{G}+Q_{L}}{A}+w_{G, j}
$$

où $C_{0}=\ldots 1,0 \ldots 1,2 \ldots$ est un paramètre de distribution, et $w_{G, j}$ une vitesse de glissement, paramètres caractéristiques tous deux de la configuration d'écoulement. Par exemple, pour l'écoulement eau-air à bouchons, à pression voisine de la pression atmosphérique, on a :

$$
C_{0}=1,2 \quad w_{G, j}=0,35 \sqrt{g D}
$$

Moyennant l'élimination de $\alpha_{G}$ au moyen de l'équation (4), le premier membre de la relation (3) divisé par $\rho_{L} g L$ peut s'écrire :

$$
\frac{1}{L} \int_{i}^{2} \frac{\dot{M}_{G}\left(1-\frac{\rho_{G}}{\rho_{L}}\right) d z}{C_{0} \dot{M}_{G}+\rho_{G}\left(C_{0} \frac{\dot{M}_{L}}{\rho_{L}}+A w_{G, j}\right)}-(1-\sigma)
$$

Dans les cas de pompage à faible profondeur (p. ex. $L<20 \mathrm{~m}$ ), on a $\rho_{G} \ll \rho_{L}$, et le terme hydrostatique moteur divisé par $\rho_{L} g L$ vaut approximativement

$$
\frac{\bar{Q}_{G}}{C_{0}\left(\bar{Q}_{G}+Q_{L}\right)+A w_{G, j}}-(1-\sigma)
$$

où $\bar{Q}_{G}$ désigne le débit volumique d'air à la pression moyenne régnant dans le tronçon diphasique. Gibson (1961) calcule le débit volumique moyen $\bar{Q}_{G}$ à partir du débit volumique en sortie $Q_{G, a}$ pour la détente isotherme par les relations :

$$
\begin{aligned}
\bar{Q}_{G}=\frac{\int_{2}^{i} Q_{G} d p}{\int_{2}^{i} d p}=\frac{\int_{2}^{i} \frac{M_{G}}{\rho_{G}} d p}{p_{i}-p_{a}}= \\
=\dot{M}_{G} R T \frac{\operatorname{Ln} \frac{p_{i}}{p_{a}}}{p_{i}-p_{a}}=Q_{G, a} \frac{p_{a}}{p_{i}-p_{a}} \operatorname{Ln} \frac{p_{i}}{p_{a}}
\end{aligned}
$$

Notons qu'en l'absence de glissement entre phases, $C_{0}=1$ et $w_{G, j}=0$, dans ce cas :

$$
\bar{\alpha}_{G}-(1-\sigma)=\frac{\bar{Q}_{G}}{\bar{Q}_{G}+Q_{L}}-(1-\sigma)
$$

Au second membre de l'équation (3), on trouve : - la perte de pression due au frottement de l'écoulement monophasique :

$$
-\int_{1}^{i}\left(\frac{d p}{d z}\right)_{f, L} d z=4 f_{L, 0} \frac{L^{\prime}}{D} \frac{\dot{M}_{L}^{2}}{2 \rho_{L} A^{2}}
$$

- la perte de pression due au frottement de l'écoulement diphasique ; une méthode consiste à la calculer par la relation:

$$
\begin{aligned}
-\int_{i}^{2}\left(\frac{d p}{d z}\right)_{f, L G} d z & = \\
& =-\int_{i}^{2}\left(1-\alpha_{G}\right)^{-1,75}\left(\frac{d p}{d z}\right)_{f, L o} d z
\end{aligned}
$$

où $(d p / d z)_{L 0}$ désigne le gradient de pression de l'écoulement du liquide circulant seul avec le débit massique de l'écoulement diphasique ; comme $\dot{M}_{G} \ll \dot{M}_{L}$, il vient :

$$
\begin{aligned}
& -\int_{i}^{2}\left(\frac{d p}{d z}\right)_{f, L G} d z= \\
& \quad=4 f_{L, 0} \frac{1}{D} \frac{\dot{M}_{L}^{2}}{2 \rho_{L} A^{2}} \int_{i}^{2}\left(1-\alpha_{G}\right)^{-1,75} d z
\end{aligned}
$$

où $\alpha_{G}$ est donné par la relation (4). Dans le cas du pompage à faible profondeur et en négligeant le glissement, il vient :

$$
\begin{aligned}
-\int_{i}^{2}\left(\frac{d p}{d z}\right)_{f, L G} & = \\
& =4 f_{L, 0} \frac{L}{D} \frac{\dot{M}_{L}^{2}}{2 \rho_{L} A^{2}}\left(\frac{Q_{L}}{\bar{Q}_{G}+Q_{L}}\right)^{-1,75}
\end{aligned}
$$

- le flux de quantité de mouvement de la phase gazeuse à la sortie

$$
\frac{\dot{M}_{G}^{2}}{A^{2} \alpha_{G, 2} \rho_{G, 2}}
$$

est généralement négligé car $\dot{M}_{G} \ll \dot{M}_{L}$.

L'équation (3) peut donc s'écrire :

$$
\begin{aligned}
& \frac{1}{L} \int_{i}^{2} \alpha_{G}\left(1-\frac{\rho_{G}}{\rho_{L}}\right) d z-(1-\sigma)= \\
& =\frac{M_{L}^{2}}{2 A^{2} \rho_{L}^{2} g L}\left\{\frac{4 f_{L, 0}}{D}\left(L^{\prime}+\int_{i}^{2} \alpha_{L}^{-1,75} d z\right)+\right. \\
& \left.\quad+2\left(\frac{1}{\alpha_{L, 2}}+\frac{\xi_{\epsilon}}{2}-1\right)\right\}
\end{aligned}
$$

Les figures 2 et 3 montrent le résultat de calculs effectués au sujet d'une installation didactique de notre laboratoire, en se basant sur l'équation ci-dessus $\left(D=27 \mathrm{~mm}, L=7,255 \mathrm{~m}, \xi_{e}=0,5, \alpha_{L, 2}=\bar{\alpha}_{L}\right)$. Ces calculs utilisent l'expression (5) du terme hydrostatique. La figure 2 suggère qu'il existe un taux de vide permettant l'obtention d'un débit liquide maximum, et que ce taux de vide augmente si le rapport d'immersion $\sigma$ diminue.

La figure 3 indique quelle est la valeur du débit d'air qui correspond au débit liquide maximum. Des débits d'air plus élevés ne conduisent qu'à une augmentation des pertes par frottement. En outre, on voit clairement qu'un débit d'air minimum est requis pour le fonctionnement de la pompe à air-lift. 


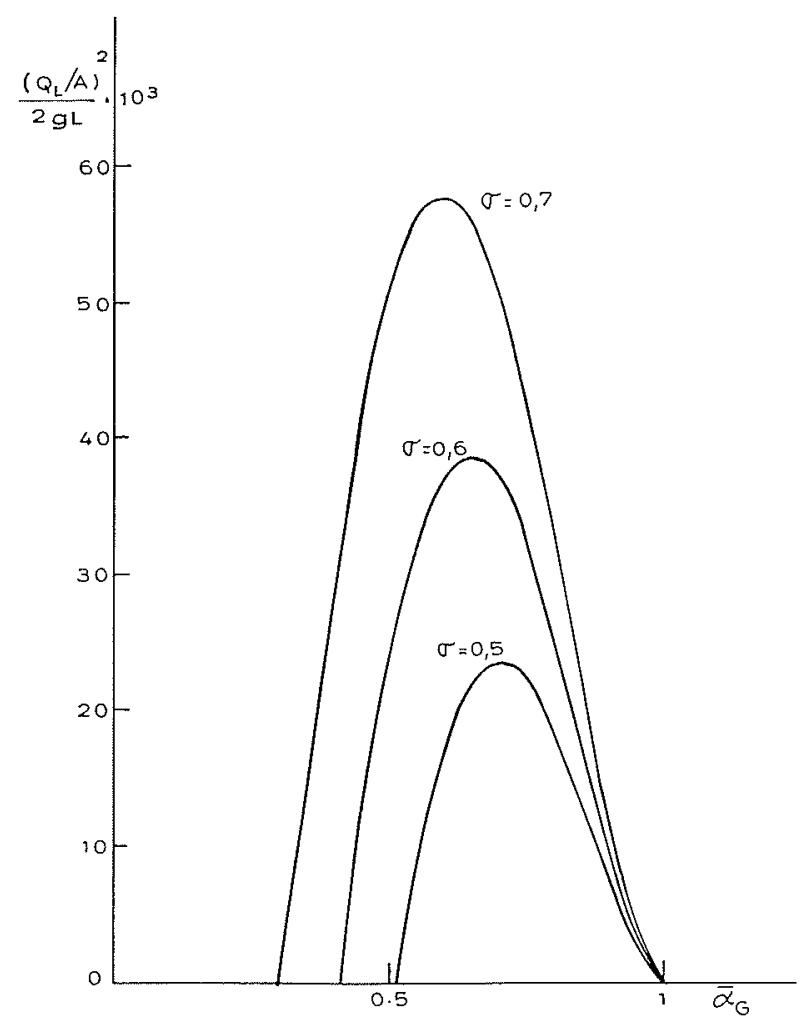

Figure 2

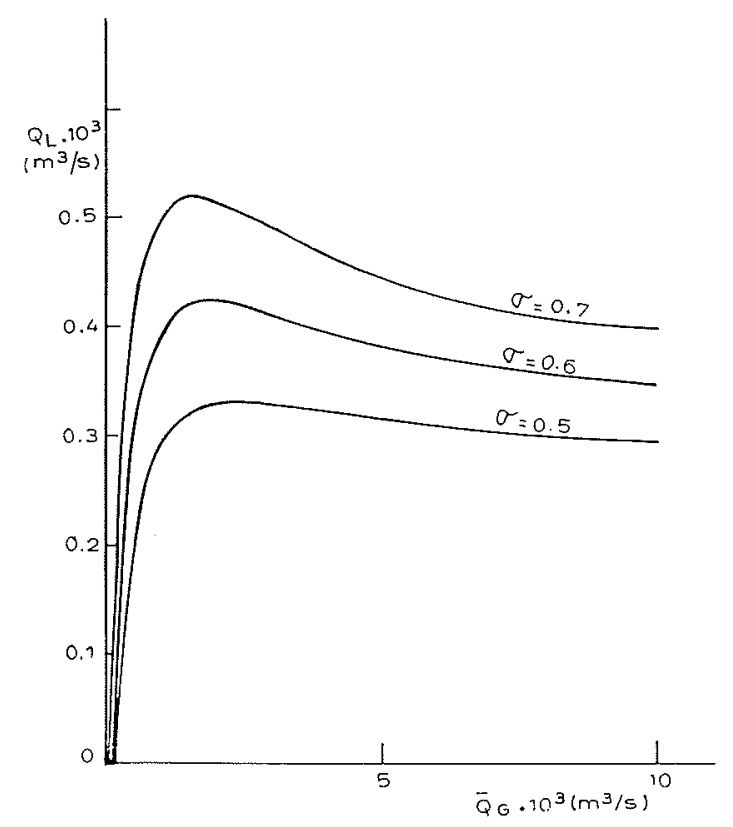

Figure 3

\section{Définition et calcul du rendement}

Le rendement $\eta$ du dispositif se définit par le rapport :

$$
\begin{aligned}
& \text { puissance théorique nécessaire } \\
& \eta \triangleq \frac{\text { pour élever le débit d'eau d'une hauteur } h_{d}}{\text { puissance à l'accouplement du compresseur }}
\end{aligned}
$$

Ce rendement peut se décomposer en deux facteurs si l'on compare le débit d'eau réellement obtenu au débit idéal qui s'écoulerait en l'absence de glissement entre phases et en l'absence de frottements. Dans ce cas, toute la puissance de compression isotherme de l'air serait convertie en puissance de pompage de l'eau. Cependant la pression à laquelle l'air devrait être injecté serait celle régnant à l'extérieur du tube au droit de l'injection, c'est-à-dire une pression plus élevée que la pression réelle d'injection tenant compte des pertes de pression à l'entrée du tube inférieur et sur sa longueur $L$ '. On peut donc écrire :

$$
\eta=\eta_{p} \eta_{C}
$$

puissance théorique nécessaire

$$
\begin{aligned}
& \eta_{P} \triangleq \frac{\text { pour l'obtention du débit réel }}{\text { puissance théorique nécessaire }} \\
& \text { puissance de compression isotherme }
\end{aligned}
$$

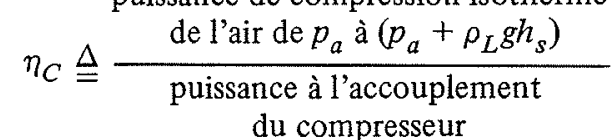

Notons que $\eta_{C}$ ne désigne pas exactement le rendement isothermique du compresseur puisque celui-ci refoule l'air à une pression qui n'est ni la pression $p_{i}$ réelle d'injection, ni celle $\left(p_{a}+\rho_{L} g h_{s}\right)$. Toutefois, dans la pratique du pompage de liquides, $\eta_{C}$ sera proche $\mathrm{du}$ rendement isothermique puisque la longueur $L^{\prime}$ est petite et que l'entrée du tube peut être profilée.

Quant au rendement $\eta_{P}$ du pompage proprement dit, il s'écrit :

$$
\eta_{P}=\frac{\rho_{L} g Q_{L} h_{d}}{\rho_{L} g Q_{L}^{\prime} h_{d}}=\frac{Q_{L}}{Q_{L}^{\prime}}
$$

si $Q_{L}^{\prime}$ désigne le débit idéal de liquide. Comme dans ce cas le second membre de l'équation (6) est nul, il vient :

$$
\bar{\alpha}_{G}^{\prime}=1-\sigma \quad \text { ou } \quad \overline{\alpha_{L}^{\prime}}=\frac{Q_{L}^{\prime}}{\bar{Q}_{G}+Q_{L}^{\prime}}=\sigma
$$

Le rendement du pompage vaut donc:

$$
\eta_{P}=\frac{Q_{L}}{Q_{L}^{\prime}}=\frac{1-\sigma}{\sigma} \frac{Q_{L}}{Q_{G}}
$$

Pour un rapport d'immersion donné, ce rendement est maximum en même temps que le rapport $Q_{L} / \bar{Q}_{G}$. La figure 3 montre que le point de rendement maximum correspond à des débits $Q_{L}$ et $\bar{Q}_{G}$ plus petits que le point de débit $Q_{L}$ maximum. Le rendement maximum augmente avec le rapport d'immersion $\sigma$ jusqu'à ce que celui-ci atteigne des valeurs de l'ordre de $\ldots 0,7 \ldots 0,8 \ldots$ et décrôit ensuite pour s'annuler évidemment lorsque $\sigma=1$

\section{Règles de conception des pompes à air-lift}

Plusieurs règles de conception se déduisent logiquement de l'analyse qui précède et des calculs qui en découlent :

\section{a) Choix du rapport d'immersion}

On a vu que le rendement $\eta_{p}$ croît si le rapport d'immersion augmente; toutefois, la pression d'injection 


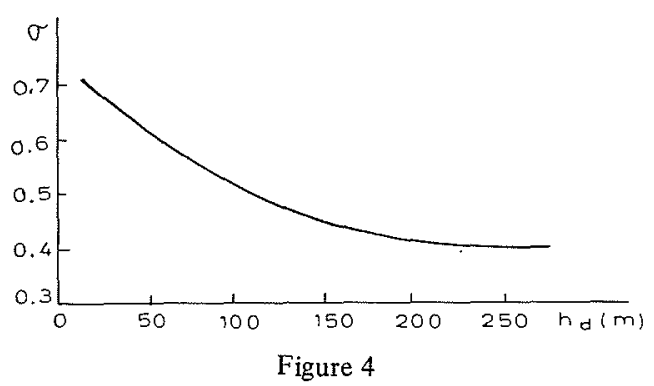

augmente elle aussi. La figure 4 inspirée de l'ouvrage de Gibbs (1971) indique le rapport d'immersion optimum en fonction de la hauteur de décharge $h_{d}$.

\section{b) Choix du diamètre du tube}

Pour limiter les pertes par frottements et par accélération, le diamètre du tube doit être choisi tel que la vitesse de l'eau ne dépasse pas $3 \mathrm{~m} / \mathrm{s}$ à l'entrée du tube. Il peut être économique d'utiliser des tubes de diamètre croissant à l'approche de la sortie, de manière à y limiter la vitesse du mélange qui se détend.

\section{c) Choix du type d'injecteur}

Le dessin de l'injecteur doit favoriser la formation de petites bulles afin d'augmenter dans la mesure du possible le taux de vide moyen $\bar{\alpha}_{G}$ qui figure dans le terme hydrostatique moteur de la pompe. Le surdimensionnement du diamètre du tube et l'utilisation de tubes de diamètre croissant à l'approche de la sortie ont un effet de même nature. Parfois on améliore le rendement en réalisant l'injection d'air par un éjecteur consistant en une pièce intérieure au tube et formant tuyère avec celui-ci.

\section{d) Choix du débit d'air}

Le fonctionnement économique a lieu à faible débit d'air. Les rendements ne dépassent toutefois que difficilement $50 \%$.

\section{Le pompage d'éléments solides par air-lift}

\section{Détermination de la relation entre le débit d'éléments solides et le débit d'air}

Comme Kato et al. (1975 a et b), intégrons l'équation de la quantité mouvement dans le volume de contrôle défini par les sections d'entrée 1 et de sortie 2 de la conduite de pompage, et la paroi de celle-ci. Il vient : $p_{1}+\alpha_{L}^{\prime} \rho_{L} w_{L, 1}^{2}+\alpha_{S}^{\prime} \rho_{S} w_{S, 1}^{2}=p_{a}+\left(\alpha_{L}^{\prime} \rho_{L}+\alpha_{S}^{\prime} \rho_{S}\right) g L^{\prime}$

$$
\begin{gathered}
+\int_{i}^{2}\left(\alpha_{G} \rho_{G}+\alpha_{L} \rho_{L}+\alpha_{S} \rho_{S}\right) g d z+\alpha_{G, 2} \rho_{G, 2} w_{G, 2}^{2} \\
+\alpha_{L, 2} \rho_{L} w_{L, 2}^{2}+\alpha_{S, 2} \rho_{S} w_{S, 2}^{2}-\int_{1}^{i}\left(\frac{d p}{d z}\right)_{f, L S} d z \\
-\int_{i}^{2}\left(\frac{d p}{d z}\right)_{f ; G L S} d z
\end{gathered}
$$

Dans cette équation, $\alpha_{L}^{\prime}$ et $\alpha_{S}^{\prime}$ désignent la fraction de la section droite occupée en moyenne respectivement par les phases liquide et solide dans le tronçon diphasique $\left(\alpha_{L}^{\prime}+\alpha_{S}^{\prime}=1\right)$, tandis que $\alpha_{G}$, $\alpha_{L}$ et $\alpha_{S}$ se rapportent au tronçon triphasique.

En supposant que la relation (2) soit encore d'application, l'équation ( 9 ) peut s'écrire :

$$
\begin{aligned}
\rho_{L} g h_{S}- & \alpha_{S}^{\prime}\left(\rho_{S}-\rho_{L}\right) g L^{\prime}- \\
& -\int_{i}^{2}\left(\alpha_{G} \rho_{G}+\alpha_{L} \rho_{L}+\alpha_{S} \rho_{S}\right) g d z \\
= & -\int_{1}^{i}\left(\frac{d p}{d z}\right)_{f, L S} d z-\int_{i}^{2}\left(\frac{d p}{d z}\right)_{f, G L S} d z \\
& +\frac{\dot{M}_{S}^{2}}{A^{2} \rho_{S}}\left(\frac{1}{\alpha_{S, 2}}-\frac{1}{\alpha_{S}^{\prime}}\right)+\frac{\dot{M}_{G}^{2}}{A^{2} \alpha_{G, 2} \rho_{G, 2}} \\
& \quad+\frac{\dot{M}_{L}^{2}}{A^{2} \rho_{L}}\left(\frac{1}{\alpha_{L, 2}}+\frac{\xi_{e}}{2 \alpha_{L}^{\prime}}-\frac{1}{\alpha_{L}^{\prime}}\right)(10)
\end{aligned}
$$

Le premier membre de cette équation est le terme hydrostatique moteur. Notons qu'il peut s'écrire :

$$
\begin{aligned}
& \int_{i}^{2} \alpha_{G}\left(\rho_{L}-\rho_{G}\right) g d z \\
& -\int_{i}^{2} \alpha_{S}\left(\rho_{S}-\rho_{L}\right) g d z-\alpha_{S}^{\prime}\left(\rho_{S}-\rho_{L}\right) g L^{\prime}-\rho_{L} g h_{d}
\end{aligned}
$$

La concentration en éléments solides dans le tronçon diphasique peut se déterminer par une relation analogue à celle (4)

$$
\frac{Q_{S}}{\alpha_{S}^{\prime} A}=C_{0}^{\prime} \frac{Q_{S}+Q_{L}}{A}-w_{S, j}
$$

où $C_{0}^{\prime}$ désigne un paramètre de distribution, et $w_{S, j}$ la vitesse de décantation des éléments solides.

Le taux de vide $\alpha_{G}$ et la concentration $\alpha_{S}$ de l'écoulement triphasique peuvent être déterminés selon Weber et Dedegil (1978) par la superposition de deux écoulements diphasiques : $L-G$ et $L-S$ selon un calcul itératif qui considère alternativement que l'écoulement $L-S$ occupe la section $\left(A-A_{G}\right)$ et que l'écoulement $L-G$ occupe $\left(A-A_{S}\right)$. Ce calcul utilise l'expression suivante pour le taux de vide de l'écoulement $L-G$ :

$$
\alpha_{G}=\frac{1}{\frac{1}{\alpha_{G, 0}}+\frac{Q_{L}}{Q_{G}}}
$$

où $\alpha_{G, 0}$ désigne le taux de vide mesuré dans une colonne de liquide stagnant ayant le diamètre de la conduite de pompage par l'élévation du niveau libre consécutive à l'injection du débit $Q_{G}$ d'air.

Kato et al. (1975a) suggèrent d'utiliser une extrapolation de la formule proposée par Akagawa pour les écoulements $L-G$ :

$$
\frac{1-\left(\alpha_{L}+\alpha_{S}\right)}{\alpha_{L}+\alpha_{S}}=c^{\prime} \frac{\left(Q_{G} / A\right)^{n}}{\left(Q_{L} / A\right)^{m}}
$$


avec les valeurs suivantes pour les constantes $c, m$ et $n$ :

\begin{tabular}{|cccc|}
\hline$\frac{Q_{L}}{A}$ & $c$ & $n$ & $m$ \\
\hline$<0,5 \mathrm{~m} / \mathrm{s}$ & 0,82 & 0,96 & 0,69 \\
$>0,5 \mathrm{~m} / \mathrm{s}$ & 0,67 & 0,78 & 0,69 \\
\hline
\end{tabular}

$\mathrm{Au}$ second membre de l'équation (10) figurent notamment les pertes de pression par frottements que Weber et Dedegil (1976) calculent à l'aide de coefficients de frottements

$-\int_{1}^{i}\left(\frac{d p}{d z}\right)_{f, L S} d z=f_{L S} \frac{4 L^{\prime}}{D} \frac{1}{2 A^{2}}\left(\frac{\dot{M}_{L}^{2}}{\alpha_{L}^{\prime} \rho_{L}}+\frac{\dot{M}_{S}^{2}}{\alpha_{S}^{\prime} \rho_{S}}\right)($

$-\int_{i}^{2}\left(\frac{d p}{d z}\right)_{f, G L S} d z$

$=f_{G L S} \int_{i}^{2} \frac{4}{D} \frac{1}{2 A^{2}}\left(\frac{\dot{M}_{G}^{2}}{\alpha_{G} \rho_{G}}+\frac{\dot{M}_{L}^{2}}{\alpha_{L} \rho_{L}}+\frac{\dot{M}_{S}^{2}}{\alpha_{S} \rho_{S}}\right) d z$

Si l'on impose une valeur du rapport $\dot{M}_{S} /\left(\dot{M}_{L}+\dot{M}_{S}\right)$ ou de $\alpha_{S}^{\prime}$, l'équation (10) fournit une relation entre $\dot{M}_{S}$ et $\dot{M}_{G}$. L'allure de ces courbes est indiquée à la figure 5 extraite de la publication de Weber et Dedegil (1976).

\section{Définition et calcul du rendement}

Le rendement du dispositif de pompage se définit par le rapport

$$
\eta \triangleq \frac{\begin{array}{l}
\text { puissance pour remonter sans } \\
\text { frottement le débit de solides }
\end{array}}{\text { puissance à l'accouplement du compresseur }}
$$

Ce rendement est le produit de deux facteurs : le rendement isothermique du compresseur $\left(\eta_{C}\right)$ et le rendement du pompage proprement dit $\left(\eta_{P}\right)$. Celui-ci s'écrit :

$$
\eta_{P}=\frac{\dot{M}_{S} g\left[\frac{\rho_{S}-\rho_{L}}{\rho_{S}}\left(L^{\prime}+h_{s}\right)+h_{d}\right]}{\dot{M}_{G} R T \operatorname{Ln} \frac{p_{r}}{p_{a}}}
$$

où la pression de refoulement $p_{r}$ vaut la pression d'injection augmentée du terme de frottement de l'écoulement d'air comprimé :

$$
p_{r}=p_{i}+(\Delta p)_{f}
$$

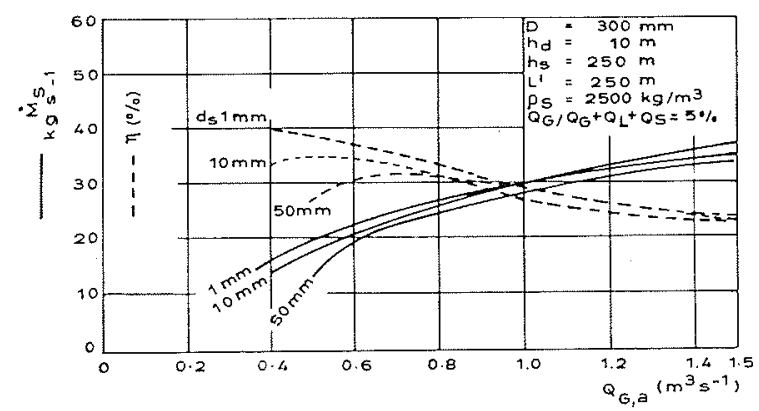

Figure 5 - (Weber et Dedegil, 1976).
La figure 5 donne des valeurs et une allure typique de ce rendement.

\section{Résultats des calculs}

\section{a) Choix du diamètre du tube}

Dans le cas du pompage des nodules, il est clair que le choix des éléments de conduites pose des problèmes de résistance des matériaux, de résistance à l'avancement etc. L'utilisation de tubes de diamètre croissant à mesure qu'on se rapproche de la surface est indispensable si l'on ne veut pas que les nodules sortent de la conduite comme des projectiles.

\section{b) Choix de la profondeur d'injection (Clauss, 1972)}

Il semble que le rendement augmente avec la profondeur d'injection pour une conduite de profil donné. Toutefois, l'accroissement de rendement s'amenuise à mesure que l'on augmente cette profondeur d'injection.

\section{c) Influence de la taille et de la concentration des élé- ments solides}

Le débit et le rendement augmentent si le diamètre équivalent des éléments solides diminue. La concentration des éléments solides dans le tronçon diphasique influence fortement le rendement. Si l'on travaille à débit de solide constant une faible concentration signifie un grand débit liquide et donc des pertes par frottements importantes, tandis qu'une concentration trop élevée signifie un accroissement exagéré du poids du mélange et donc, à nouveau, une perte de rendement.

\section{Reférences bibliographiques}

CLAUSS G. -- Some Investigations into air-lift systems for mineral recovery in ocean mining, WODCON IV Transactions, 1972.

GIBBS Ch. - Compressed Air and Gas Data. Ingersoll-Rand Company, 2d Edition, 1971.

GIBSON. - Hydraulics and its applications, Constable and $\mathrm{C}^{\circ}$ Ltd, London, 5 th Edition, 814 p., 1961.

GRABOW G. - Lift-Verfahren mit Auftriebselementen zur Förderung von Manganknollen aus grossen Mevrestiefen, Seewirtschaft, 9, 5, 295-299, 1977.

KATO H., TAMIYA, S. and MIYAZAWA T. - A study of an air-lift mump for solid particles and its application to marine engineering. 2nd Symposium on Jet Pumps \& Ejectors and Gas Lift Techniques, March 1975, G3, 37-49, $1975 \mathrm{a}$.

KATO H., MIYAZAWA T., TIMAYA $S$ and IWASAKI, T. - A study of an air-lift pump for solid particles, Bull. JSME, I8, $117,286-294,1975 \mathrm{~b}$.

TODOROKI I., SATO Y. and HONDA T. - Performance of AirLift Pump, Bull. JSME, 16, 94, 733-741, 1973.

WEBER M. and DEDEGIL Y. - Transport of solids according to the air-lift principle, Fourth International Conference on the Hydraulic Transport of Solids in Pipes, May 1976, H1, $1-24,1976$

WEBER M., DEDEGIL Y., and FELDLE G. - New experimental results regarding extreme operating conditions in air lifting and vertical hydraulic transport of solids according to the jet lift principle and its applicability to deep-sea mining. Fifth International Conference on the Hydraulic Transport of Solids in Pipes, BHRA Fluid Engineering, Cranfield, Bedford U.K., May 1978, F7, 1-26, 1978.

ZUBER N. and FINDLAY J.A. - Average volumetric concentration in two-phase systems, $J$. Heat Transfer, 453-468, 1965 . 


\section{Discussion}

M. le Président. - Merci beaucoup M. le Professeur pour cet intéressant exposé. Qui demande la parole?

M. MONDIN. - Est-ce que vous avez vérifié la similitude de Froude par rapport au diamètre?

M. GIOT. - Non.

M. MONDIN, - Nous l'avons fait. Cela marche jusqu'à un certain diamètre.

M. FITREMANN. - En général le minimum des courbes de pression c'est-à-dire l'optimum de la concentration en gaz se produit pour des écoulements en poche.

Est-ce cette configuration que vous avez observée?

M. GIOT. - Lorsque le pompage doit se faire à l'échelle de millions de tonnes par an, les conduites sont de grand diamètre, par exemple 400 à $500 \mathrm{~mm}$. Dans ce cas, on n'observe pas d'écoulement à bouchons, mais plutôt des écoulements fortement turbulents, constitués de grosses bulles qui sont fortement remuées. Il n'y a pas de configuration d'écoulement très caractéristique.

M. DUPORT. - Est-ce qu'on a vérifié le fonctionnement triphasique dans la zone supérieure de la conduite à l'endroit où le taux de vide est grand?

M. GIOT. - Oui, puisqu'on a réussi à faire fonctionner cette pompe en vraie grandeur dans l'Océan Pacifique... pas en vraie grandeur de débit, mais à $4500 \mathrm{~m}$ de profondeur.

$M . D U P O R T$. - Avec le triphasique...?

M. MONDIN. - Avec une section variable?

M. GIOT. - Oui, il est exclu d'avoir une section constante, ne serait-ce que pour des questions mécaniques.

M. JACQUEMIN. - Je voulais signaler qu'entre 1972 et 1975 une équipe a effectué au C.E.A. des essais de pompage par airlift. On a mesuré l'influence de divers paramètres, y compris l'inclinaison du tube.

M. GIOT. - Jusqu'à quel angle êtes-vous allés?

M. JACQUEMIN. - Jusqu'à $45^{\circ}$ en écoulement diphasique air-eau. Le débit d'eau est alors réduit mais on a encore $Q_{45^{\circ}} \cong 0,5 Q_{0}{ }^{\circ}$. En écoulement triphasique une inclinaison de 10 a $15^{\circ}$ est sans influence notable.

Les essais effectués jusqu'à une profondeur de $200 \mathrm{~m}$ $\left(\phi_{\text {tube }}=0,2 \mathrm{~m}\right)$ montrent des courbes de débit solide très plates.
Lorsque les particules solides sont très petites on a mesuré aux concentrations élevées $(C \sim 0,20)$ une sorte de "renforcement" du débit solide, mis sur le compte d'un effet bloquant la ségrégation de l'air.

Nous avons par ailleurs obtenu des résultats tout à fait analogues à ceux qui viennent d'être présentés par M. GIOT.

M. MONDIN. - En triphasique on a un paramètre libre qu'on peut utiliser pour faire une optimisation des courbes que vous avez données. C'était à concentration donnée, ou à débit solide donné ?

On peut imaginer une régulation qui, en fonction de la densité de nodules sur le fond, et donc d'une information recueillie au fond, permettrait de travailler près de l'optimum.

M. GIOT. - Je crois que malheureusement on n'en est pas encore là. Par contre le point dont on est préoccupé, c'est de faire en sorte de ne pas avoir une concentration trop forte dans la tuyauterie et d'être capable de vider cette tuyauterie au cas où l'on risquerait un bouchage. On n'en est pas encore à faire la régulation fine, comme il serait souhaitable.

De toute façon il ne faut pas s'imaginer que les conditions de sol soient uniformes. Le relief n'est pas uniforme; la vitesse d'avance n'est pas uniforme ; la concentration ne l'est pas non plus. Il y a toujours des variations de concentration à travers la tuyauterie.

M. le Président. - Comment se situe la méthode air-lift par rapport aux méthodes purement mécaniques de ramassage des nodules du point de vue économique?

M. GIOT. - Mes amis français seraient plus à même que moi de répondre à cette question, puisque le système à godets a été imaginé par eux.

A mon avis, un procédé hydraulique est plus facile à mettre en œuvre. En tout cas, ce que je peux dire, c'est que le problème de la pompe à air-lift elle-même est vraiment le problème le plus facile à résoudre dans l'ensemble des problèmes techniques et autres qui se posent à propos de la récupération des nodules.

M. le Président. - Pour ce qui concerne les procédés mécaniques j'en signale un autre qui a été imaginé par le C.E.A. : il implique une noria de véhicules autonomes télé-commandés. Je ne sais pas si vous l'avez pris en considération dans votre comparaison...

M. GIOT. - Non.

M. le Président. - Je vous remercie beaucoup M. le Professeur.

\begin{tabular}{|c|c|}
\hline \multicolumn{2}{|c|}{ Abstract } \\
\hline \multicolumn{2}{|c|}{ Air-lift pumping and raising of polymetal nodules from the bottom of the sea } \\
\hline $\begin{array}{l}\text { Basic principles for the design of air-lift pumps and their } \\
\text { applications to the pumping of polymetallic nodules from the } \\
\text { floor of the oceans are presented in two sections. } \\
\text { The first section discusses the pumping of liquids. The rela- } \\
\text { tionship between the liquid and gas flow-rates is deduced from } \\
\text { the momentum equation of the mix ture and by using the } \\
\text { Zuber and Findlay expression for the void fraction. The total } \\
\text { efficiency of the pump is divided into two factors : one is the } \\
\text { isothermal efficiency of the compressor, whereas the other is } \\
\text { related to the two-phase flow in the pipe. Results of calcula- }\end{array}$ & $\begin{array}{l}\text { tions are presented and general design rules are deduced } \\
\text { concerning submergence ratio, tube diameter, type of injector } \\
\text { and air flow-rate. } \\
\text { In the second section, on the pumping of solid elements by } \\
\text { means of the air-lift pump, the momentum equation of the } \\
\text { three-phase flow is written, and expressions are given for both } \\
\text { void fraction and friction pressure drop. Typical results of the } \\
\text { solid versus gas flow-rate and efficiency versus gas flow-rate } \\
\text { calculations are presented. }\end{array}$ \\
\hline
\end{tabular}

